\title{
AVALIAÇÃO DO TIPO DE DIAMANTE DE BENZEL EM PACIENTES PORTADORES DE MIELOPATIA CERVICAL
}

\author{
EVALUATION OF THE TYPE OF BENZEL'S DIAMOND IN PATIENTS \\ WITH CERVICAL MYELOPATHY
}

\author{
EVALUACIÓN DEL TIPO DE DIAMANTE DE BENZEL EN PACIENTES \\ CON MIELOPATIA CERVICAL
}

Rodrigo Rezende ${ }^{1}$, Igor Machado Cardoso² ${ }^{2}$ Charbel Jacob Junior ${ }^{3}$, José Lucas Batista Junior ${ }^{4}$, Thiago Ferreira Nunes Pereira ${ }^{5}$

\begin{abstract}
RESUMO
Objetivo: Difundir o método de Benzel para aferição da angulação cervical e quantificar a prevalência de cada tipo de diamante de Benzel em 30 pacientes portadores de mielopatia cervical com indicação cirúrgica. Métodos: Estudo retrospectivo de pacientes portadores de mielopatia cervical submetidos a tratamento cirúrgico. Foram analisadas radiografias em perfil da coluna cervical onde se aferiram os tipos diamante de Benzel usando técnicas descritas pelo próprio Benzel. Excluímos do estudo aqueles pacientes que desenvolveram a doença após traumas, que foram submetidos reoperações ou aqueles que não forneceram radiografias em perfil adequadas para aferição. Resultados: Foram avaliados 30 pacientes, sendo 25 deles do sexo masculino, com idade entre 30 e 74 anos (média de 52,4 anos). Após a realização das medidas foram encontrados 24 pacientes com diamantes do tipo A (80\%), 2 do tipo B (6,7\%) e 4 tipo C (13,3\%). Conclusão: O diamante de Benzel tipo "A" foi encontrado em $80 \%$ dos pacientes portadores de mielopatia cervical no período pré-operatório.
\end{abstract}

Descritores: Coluna vertebral; Doenças da medula espinal; Cifose; Mielopatia cervical.

\begin{abstract}
Objective: Diffuse the method of Benzel for calibration of cervical angulation and to assess the prevalence of each type of Benzel's diamond between 30 patients with cervical myelopathy with an indication for surgical treatment. Methods: A retrospective study of patients with cervical myelopathy treated surgically. We analyzed lateral radiographs of cervical spine where the diamonds were measured using the techniques described by the author himself. We excluded patients who developed disease following trauma, who underwent reoperations or those who have not provided adequate lateral radiographs for measurement. Results: We evaluated 30 patients, of whom 25 were male, aged between 30 and 74 years (mean 52.4 years). After completion of the measures 24 patients were found with diamond type $A(80 \%), 2$ with type B (6.7\%) and 4 with type C (13.3\%). Conclusion: The Benzel diamond "A" was found in $80 \%$ of patients with cervical myelopathy in the preoperative evaluation.
\end{abstract}

Keywords: Spine; Spinal cord diseases; Kyphosis; Cervical myelopathy

RESUMEN

Objetivo: Difundir el método de Benzel para la medición del ángulo cervical y cuantificar la prevalencia de cada tipo de diamante Benzel en 30 pacientes portadores de mielopatía cervical con indicación quirúrgica. Métodos: Estudio retrospectivo de los pacientes con mielopatía cervical sometidos a tratamiento quirúrgico. Se analizaron las radiografías de perfil de la columna cervical, donde se midieron los tipos de diamante utilizando las técnicas descritas por el mismo Benzel. Se excluyeron del estudio los pacientes que desarrollaron la enfermedad después de trauma, que fueron sometidos a reoperaciones o los que no han presentado adecuadas radiografías de perfil para la medición. Resultados: Se evaluaron 30 pacientes entre los cuales 25 eran del sexo masculino, con edades comprendidas entre 30 y 74 años (promedio de 52,4 años). Después de la realización de las medidas se encontraron 24 pacientes con diamante del tipo A (80\%), 2 con tipo B (6,7\%) y 4 con tipo C (13,3\%). Conclusión: El diamante de Benzel del tipo "A" se encuentra en el 80\% de los pacientes con mielopatía cervical en el período preoperatorio.

Descriptores: Columna vertebral; Enfermedades de la médula espinal; Cifosis, Mielopatía cervical

\section{INTRODUÇÃO}

A mielopatia espondilolítica é uma disfunção da medula espinhal relacionada à degeneração típica do envelhecimento caracterizada por alterações do motoneurônio superior, que leva a um quadro clínico de hiperreflexia, distúrbios da marcha e propriocepção'. A prevalência exata é desconhecida, mas sabe-se que acomete mais o sexo masculino, em pacientes acima de 55 anos de idade ${ }^{2,3}$.

O tratamento da mielopatia cervical na maioria das vezes é cirúr- gico, porém, a decisão de se realizar uma descompressão por via anterior, posterior ou combinada continua sendo foco de diversas discussões, devendo ser levados em consideração diversos fatores como idade do paciente, principal local de compressão, número de níveis acometidos e a presença ou não de cifose cervical ${ }^{4}$

O método para cálculo da angulação da cifose cervical mais usado é o definido por $\mathrm{Cobb}^{5}$, que muitas vezes se torna impossível de ser realizado pela dificuldade de se visibilizar o platô superior

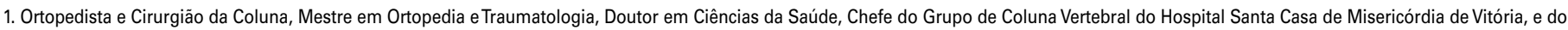
Vila Velha Hospital -ES, Brasil.

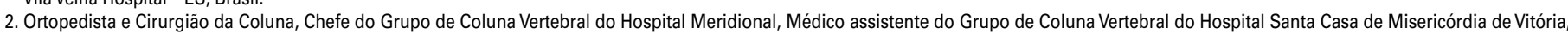
ES, Brasil.

3. Ortopedista Especialista em Cirurgia da Coluna Vertebral, Médico Assistente da Santa Casa de Misericórdia de Vitória - ES, Brasil.

4. Ortopedista Especialista Em Cirurgia Da Coluna Vertebral, Médico Assistente da Santa Casa de Misericórdia de Vitória - ES, Brasil.

5. Ortopedista Especialista em Cirurgia da Coluna Vértebra - ES, Brasil.

Trabalho realizado no hospital da Santa Casa de Misericórdia de Vitória - Vitória, ES. Brasil.

Correspondência:Thiago Ferreira Nunes Pereira, AV. Hugo Musso 2042, Ap. 503. Torre Nice- 29101785 -Vila Velha - ES. Brasil. t.f.n.p@ hotmail.com/ grupodecoluna@santacasavitoria.org

Recebido em 31/01/2012, aceito em 22/02/2012. 
de C2 e C7. Outro método de aferição da cifose foi definido por Benzel $^{6}$, sendo descrito como técnica do diamante de Benzel no qual, através de uma radiografia simples em perfil, se consegue detectar a presença ou não de cifose cervical.

Sabemos que a mielopatia cervical é uma doença grave e quando não diagnosticada precocemente e com maior precisão possível pode levar a danos importantes e até mesmo irreversíveis ${ }^{7}$ aos pacientes. Por este motivo, julgamos que é de extrema importância realizar, dentre outros critérios já citados, uma adequada avaliação da angulação cervical, pois ela pode ser decisiva no momento de se indicar a técnica cirúrgica a ser utilizada.

Realizamos este estudo com o objetivo de difundir a técnica de Benzel e avaliar qual o tipo de diamante de Benzel mais prevalente em pacientes portadores de mielopatia cervical.

\section{MATERIAIS E MÉTODOS}

Estudo retrospectivo de trinta pacientes portadores de mielopatia cervical submetidos a tratamento cirúrgico atendidos no grupo de coluna do Serviço de Ortopedia e Traumatologia da Santa Casa de Vitória e Hospital Vila Velha no período de janeiro de 2008 a dezembro 2010.

Foram incluídos no estudo todos os pacientes portadores de mielopatia cervical de origem degenerativa num total de 30 pacientes, sendo 25 deles do sexo masculino, com idade entre 30 e 74 anos (média de 52,4 anos). Foram excluídos os pacientes que desenvolveram mielopatia cervical após traumas, reoperações, tumores ou aqueles que não forneceram radiografias em perfil adequadas para a obtenção dos dados deste trabalho.

A avaliação de imagem foi feita a partir de exames de radiografias simples em perfil da coluna cervical que exibissem os corpos vertebrais de C2 a C7 e que possibilitassem a confecção do diamante de Benzel, sendo estas medidas feitas para cada indivíduo pesquisado neste trabalho.

O diamante de Benzel ${ }^{6,8}$ foi descrito pelo próprio autor como método de detecção de cifose cervical e, segundo Benzel, existem três possíveis formas do diamante que variam de acordo com o posicionamento dos corpos vertebrais de C3 a C6 dentro de uma figura losangular confeccionada entre C2 e C7. Primeiramente, é traçada uma linha entre um ponto situado no aspecto dorso caudal de C2 e dorso caudal de C7 (Figura 1A). Posteriormente, afere-se o ponto médio desta linha e a partir deste ponto são traçados mais dois outros pontos, um $2 \mathrm{~mm}$ anterior ao ponto médio e um $2 \mathrm{~mm}$ posterior, para se traçar outra reta perpendicular a primeira, de $4 \mathrm{~mm}$ de largura (Figura 1B). O próximo passo é ligar os quatro pontos marcados, formando uma figura losangular, o chamado diamante de Benzel (Figura 1C).

As formas do diamante de Benzel são classificadas como A, B ou C (Figura 2), sendo que no tipo A consiste numa figura losangular posterior aos corpos vertebrais, o que caracteriza uma curva fisiológica normal ou, segundo o autor, uma "lordose efetiva". No tipo $B$, os corpos vertebrais se encontram totalmente sobrepostos
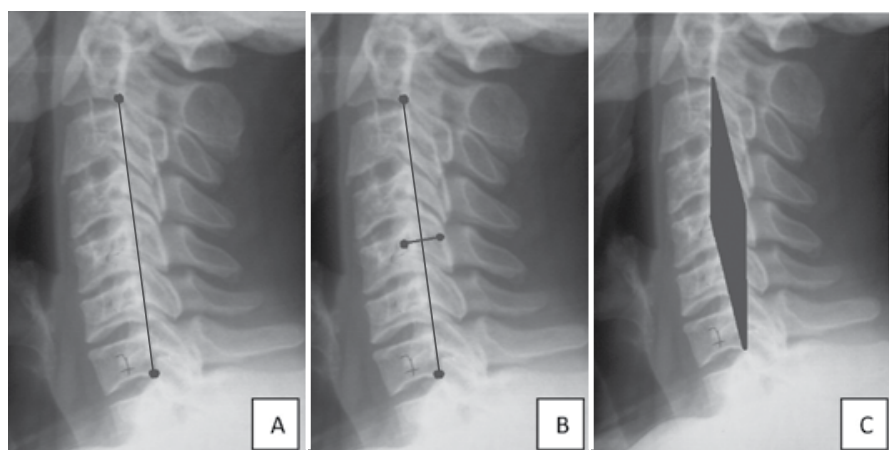

Figura 1. (A) Pontos de referências em C2 e C7. (B) Pontos $2 \mathrm{~mm}$ anterior e posterior a linha principal. (C) Após interligar os pontos temos o Diamante de Benzel. à figura losangular caracterizando uma cifose cervical verdadeira ou "cifose efetiva". Já no tipo $\mathrm{C}$, há uma cobertura parcial dos corpos vertebrais dentro da figura losangular desenhada, figura designada pelo autor como "zona cinzenta" onde não há certeza se há ou não cifose da coluna cervical (Figura 2)

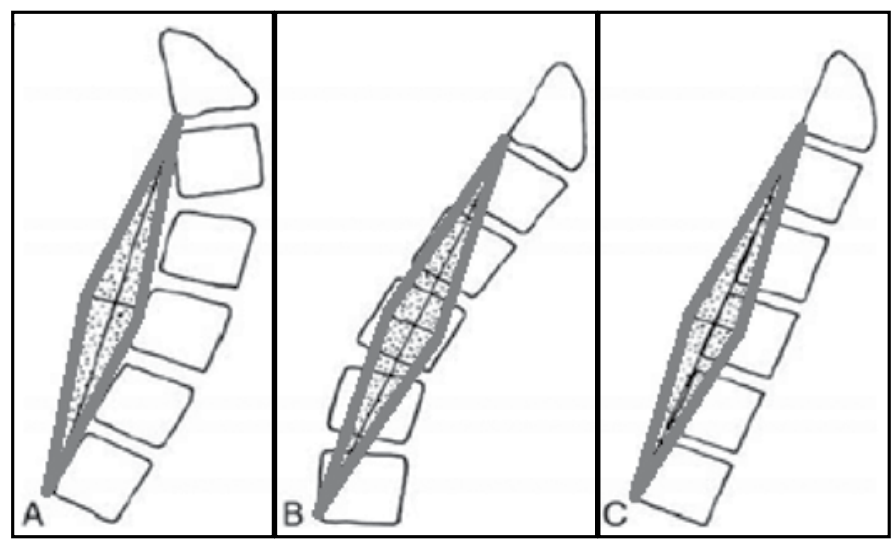

Figura 2. Tipos de diamantes: (A) - normal (B) - cifose (C)- zona cinzenta.

\section{RESULTADOS}

Os tipos de diamante de Benzel foram distribuídos percentualmente dentro da amostra, definindo a prevalência de cada tipo. Encontramos 24 pacientes com diamante tipo A (80\%), ou seja, sem presença de cifose segundo o método empregado, dois pacientes com diamante tipo B $(6,7 \%)$, ou seja, com presença de cifose verdadeira na coluna cervical segundo o método e quatro pacientes com diamante do tipo C (13,7\%) sendo estes chamados de "zona cinzenta" onde não se pode afirmar se há ou não cifotização verdadeira da coluna cervical (Tabela 1).

Tabela 1. Resultados da amostra.

\begin{tabular}{c|c|c}
\hline Tipo de diamante & $\mathbf{N}^{0}$ de pacientes & Pocerntagem (\%) \\
\hline A & 24 & 80 \\
\hline B & 2 & 6,7 \\
\hline C & 4 & 13,3 \\
\hline Total & 30 & 100 \\
\hline
\end{tabular}

\section{DISCUSSÃO}

O método de Benzel é descrito na literatura como meio de aferição da cifose da coluna cervical e constitui sem dúvida uma ferramenta diagnóstica importante, de fácil aferição, rápida e não acrescenta nenhum custo adicional além da rotina radiográfica pré-operatória já estabelecida na literatura. Através dele é possível determinar a presença ou não de cifose cervical ${ }^{6}$.

A escolha da via de acesso anterior ou posterior para o tratamento da mielopatia cervical é influenciada por diversos fatores ${ }^{9}$, entre eles o número de níveis acometidos, calcificação do ligamento longitudinal posterior, estenose cervical congênita e a presença de cifose cervical, sendo esta uma contra-indicação relativa para se realizar a via posterior cervical ${ }^{4,6,10,11}$.

Nosso trabalho tem o objetivo de difundir uma técnica de aferição da angulação da coluna cervical em pacientes portadores de mielopatia cervical e investigar a presença e incidência da cifose cervical nestes pacientes e se justifica no fato da existência de muitos relatos na literatura nos quais à mielopatia cervical de origem degenerativa leva a maior predisposição a apresentar retificação ou inversão da lordose cervical ${ }^{11-14}$.

Estas alterações angulares podem ocasionar a piora da mielopatia cervical devido ao aumento do estresse mecânico ${ }^{11}$ na face anterior da medula espinhal com uma progressão da cifose, 
levando à falha de enchimento das artérias nutridoras do segmento medular, principalmente em momentos de força em flexão, à redução de fornecimento de sangue e à progressão da lesão para mielomalácia e atrofia da medula espinhal; consequentemente a um pior prognóstico de recuperação neurológica ${ }^{12,15,16}$ pós-operatória.

Por estes motivos, a presença de cifose cervical associada a mielopatia é indicativo de cirurgia utilizando a via de acesso anterior ${ }^{13}$ para restabelecer a lordose fisiológica da coluna cervical, sendo este procedimento adotado pela maioria dos cirurgiões ${ }^{17}$.

Os dados apurados em nosso trabalho mostraram que apesar da presença de mielopatia com indicação cirúrgica, 80\% dos pacientes não possuíam uma coluna cervical cifotizada segundo o diamante de Benzel e a presença de cifose verdadeira foi constatada em apenas 6,7\% dos pacientes.

Estes dados não puderam ser confrontados com outros trabaIhos, pois não encontramos na literatura pesquisas semelhantes utilizando o método de Benzel na avaliação radiológica dos pacien- tes com mielopatia cervical. Contudo, há trabalhos na literatura que demonstram, pelo método de Cobb, que pacientes portadores de mielopatia cervical possuem baixa prevalência de cifose ${ }^{13}$, concordando com os dados de nosso trabalho.

A aferição da angulação cervical pelo método de Cobb se torna muitas vezes difícil devido ao padrão radiográfico, portanto o método de Benzel consiste em uma boa ferramenta para auxiliar a detecção da presença de cifose cervical, pois é um método de fácil aplicabilidade e de execução bem definida pelo estudo de Benzel, podendo ser uma alternativa ao método de Cobb para avaliação da coluna cervical.

Nossos resultados mostram que na análise global da curvatura cervical existe predomínio do eixo lordótico cervical (diamante tipo "A").

\section{CONCLUSÃO}

O diamante de Benzel Tipo A foi encontrado em $80 \%$ dos pacientes portadores de mielopatia cervical no período pré-operatório.

\section{REFERÊNCIAS}

1. Young WF. Cervical spondylotic myelopathy: a common cause of spinal cord dysfunction in older persons. Am Fam Physician. 2000;62(5):1064-70, 1073.

2. Crandall PH, Gregoriuis FK. Long-term follow-up of surgical treatment of cervical Spondylotic myelopathy. Spine. 1977;2:139-46.

3. Orr RD, Zdeblick TA. Cervical spondylotic myelopathy. Approaches to surgical treatment. Clin Orthop Relat Res. 1999;(359):58-66.

4. Suda K, Abumi K, Ito M, Shono Y, Kaneda K, Fujiya M. Local kyphosis reduces surgical outcomes of expansive open-door laminoplasty for cervical spondylotic myelopathy. Spine (Phila Pa 1976). 2003;28(12):1258-62.

5. Cobb JR. Outline for the study of scoliosis. Instr Course Lect. 1948;5:261-7.

6. Benzel EC. Biomechanics of cervical spine surgery for tumor and degenerative diseases. Neurol Med Chir (Tokyo). 1997;37(8):583-93.

7. Cheung WY, Arvinte D, Wong YW, Luk KD, Cheung KM. Neurological recovery after surgical decompression in patients with cervical spondylotic myelopathy a prospective study. Int Orthop. 2008;32(2):273-8.

8. Shedid D, Benzel EC. Cervical spondylosis anatomy: pathophysiology and biomechanics. Neurosurgery. 2007;60(1 Suppl 1):S7-13. 17204889.

9. Rao RD, Gourab K, David KS. Operative treatment of cervical spondylotic myelopathy. J Bone Joint Surg Am. 2006;88(7):1619-40. 16818991.

10. O'Shaughnessy BA, Liu JC, Hsieh PC, KoskiTR, Ganju A, Ondra SL. Surgical treatment

of fixed cervical kyphosis with myelopathy Spine (Phila Pa 1976). 2008:33(7):771-8.

11. Masini M, Maranhão V. Experimental determination of the effect of progressive sharp-angle spinal deformity on the spinal cord. Eur Spine J. 1997;6(2):89-92.

12. Breig A, Turnbull I, Hassler O. Effects of mechanical stresses on the spinal cord in cervical spondylosis. A study on fresh cadaver material. J Neurosurg. 1966;25(1):45-56.

13. Herman JM, Sonntag VK. Cervical corpectomy and plate fixation for postlaminectomy kyphosis. J Neurosurg. 1994;80(6):963-70

14. Iwasaki M, Yamamoto T, Miyauchi A, Amano K, Yonenobu K. Cervical kyphosis: predictive factors for progression of kyphosis and myelopathy. Spine (Phila Pa 1976). 2002;27(13):1419-25.

15. Breig A, el-Nadi AF. Biomechanics of the cervical spinal cord. Relief of contact pressure on and overstretching of the spinal cord. Acta Radiol Diagn (Stockh). 1966;4(6):602-24.

16. Naderi S, Ozgen S, Pamir MN, Ozek MM, Erzen C. Cervical spondylotic myelopathy: surgical results and factors affecting prognosis. Neurosurgery. 1998:43(1):43-9.

17. Sevki K, MehmetT, UfukT, Azmi H, Mercan S, Erkal B. Results of surgical treatment for degenerative cervical myelopathy: anterior cervical corpectomy and stabilization. Spine (Phila Pa 1976). 2004;29(22):2493-500. 